Hirls, G. H. the late \& Spurr Errol D. (1952). J. gen. Microbiol. 6, 64-73.

\title{
The Effect of Temperature on the Nutritional Require- ments of Pasteurella pestis
}

\author{
By The Late G. M. HILls and ERROL D. SPURR
}

Microbiological Research Department, Experimental Station, Porton, Wiltshire

\begin{abstract}
SUMMARY: The nutrition of three virulent and three avirulent strains of Pasteurella pestis has been studied at temperatures between 23 and $37^{\circ}$ on a basal medium containing glucose, ammonium and other inorganic salts. The organism has considerable synthetic powers and the distinction between essential nutrients and non-specific stimulants of growth is not always definite. At $32^{\circ}$ and below, the optimal medium contained phenylalanine, valine, isoleucine, cysteine, methionine and haemin. Five out of the six strains utilized leucine in place of valine, but the maximum count was then delayed. At $36^{\circ}$ the optimal medium contained in addition, alanine, leucine, serine, threonine, biotin and pantothenate. Omission of alanine or leucine delayed growth without reducing the maximum population. When biotin and pantothenate were omitted the organism required a mixture of twenty amino-acids.
\end{abstract}

The nutrition of Pasteurella pestis has been studied with conflicting results by Rao (1939, 1940b), Berkman (1942), Doudoroff (1943) and Herbert (1949). Rao (1939), using large washed inocula (c. $10^{7}$ cells $/ \mathrm{ml}$.) at $27^{\circ}$, claimed that the only amino-acids for which there was a specific requirement were phenylalanine, proline and cystine, but that glycine was stimulatory and at least two of the four comprising alanine, leucine, lysine and arginine were also required. Metabolic experiments (Rao, 1940 a) showed that arginine and lysine were not appreciably oxidized and these were then omitted from media used for investigating the stimulatory effects of some of the then-known growth factors (Rao, 1940 b), but other amino-acids oxidizable by the organism were also added, viz. valine, isoleucine, methionine, serine, tyrosine and glutamate. Doudoroff (1943), working at $29^{\circ}$, claimed that only cystine and phenylalanine were essential on first transfer from blood agar, although proline stimulated two pathogenic strains. A non-pathogenic strain was able to utilize thiosulphate, sulphite, thiolacetic acid or homocystine, but not methionine, in place of cystine. Large inocula were necessary, however. Berkman (1942), using an inoculum of $1-2 \times 10^{4} \mathrm{cells} / \mathrm{ml}$. and a temperature of $37^{\circ}$, found that four out of five strains grew in 1-2 days on a mixture of $18 \alpha$-amino-acids or a gelatin hydrolysate medium, but the fifth strain failed to grow under these conditions. Using a similar medium containing $20 \alpha$-aminoacids, Herbert (1949), also working at $37^{\circ}$, found growth to be irregular even with as large an inoculum as $10^{6}$ organisms $/ \mathrm{ml}$., but the addition of haemin led to consistent growth from as few as $10 \mathrm{cells} / \mathrm{ml}$.

Rao (1939) and Doudoroff (1943), working below $30^{\circ}$ in the neighbourhood of the recognized optimum temperature, agree in claiming simpler nutritional requirements than those observed at $37^{\circ}$ by Berkman (1942) and Herbert (1949). The observations of the two former authors are of little value, however, owing to the use of large inocula. It was considered desirable therefore to 
re-investigate the nutritional requirements of Past. pestis at $27-29^{\circ}$ using small inocula. Although, in general, it may be of little value to study the nutritional requirements of an organism at a temperature removed from the optimum, the problem of the nutrition of Past. pestis in the neighbourhood of $37^{\circ}$ was also studied further on account of the virulence of the organism for some warm-blooded animals and because growth at this temperature is of importance for the production of the prophylactic envelope antigen of Schütze $(1932,1939)$.

\section{METHODS}

In general, the methods were those of Herbert (1949) modified as follows.

Glassware. After the usual chemical cleaning in chromic-sulphuric acid, hot where possible, glassware was rinsed freely with tap water and distilled water, soaked in distilled water for several hours to remove adsorbed chromium and finally rinsed several times in glass distilled water.

Amino-acid media. Constituents were sterilized separately (hydroxy- and sulphur-containing compounds by Seitz-filtration) and mixed aseptically just before use. Glucose (Seitz-filtered) was present in the media for all experiments reported, but to avoid excessive development of acidity during growth the concentration was decreased to $5 \mathrm{~mm}$. For the same reason, the ammonium sulphate concentration was decreased to $2 \mathrm{~mm}$ in most experiments where it was used. In some experiments it was replaced by ammonium lactate, 4 mM., until this was found to be disadvantageous rather than beneficial. The concentrations of amino-acids were (mM.): glycine, $1 \cdot 4-2 \cdot 0$; DL-alanine, $1 \cdot 0-2 \cdot 0$; DL-valine, 1.0; DL-leucine, 1.0; DL-isoleucine, 1.0; DL-norleucine, 1.0-2.0; DL-serine, 1.0; DL-threonine, 1.0; DL-methionine, 0.4-1.0; DL-homocystine (S), 0.4; DL-cysteine, 0.4-0.5; DL-cystine (S), 0.4; DL-aspartic acid, 1.0-2.0; DL-glutamic acid, 8-10; DL-lysine, 1.4; L-arginine, 0.7 ; L-histidine, 0.7-1.0; DL-proline, 1.4-2.0; L-hydroxyproline, 0.7; DL-phenylalanine, 4.0; DL-tyrosine, $0 \cdot 7$; and DL-tryptophan, $0 \cdot 3$ [(S) signifies a synthetic preparation]. The concentrations of other growth factors were: biotin, $0.005 \mu \mathrm{M}$; folic acid, calcium pantothenate, riboflavin and aneurin 1.0 $\mu \mathrm{M}$.; pyridoxin, $2 \cdot 0 \mu \mathrm{M} . ; p$-aminobenzoate and nicotinamide, $5 \mu \mathrm{M}$.; choline, haemin, inositol and pimelic acid, $10 \mu \mathrm{M}$. ; adenine, guanine, xanthine, cytosine, thymine, uracil and glutamine, $50 \mu \mathrm{M}$.; glutathione, $200 \mu \mathrm{M}$. The concentrations of the cations were: $\mathrm{Fe}^{++}$ (as $\mathrm{FeSO}_{4} .\left(\mathrm{NH}_{2}\right)_{2} \mathrm{SO}_{4} \cdot 6 \mathrm{H}_{2} \mathrm{O}$ ) $0.5 \mu \mathrm{M}$.; $\mathrm{Mg}^{++}$(as $\mathrm{MgSO}_{4}$ ), $200 \mu \mathrm{M}$.; Ca ${ }^{++}$(as $\mathrm{CaCl}_{2}$ ), usually $10 \mu \mathrm{M}$. but sometimes increased to $100 \mu \mathrm{M}$., when $1 \mathrm{~mm}$. sodium citrate was added to hold the metal in solution; $\mathrm{Cu}^{++}$(as $\mathrm{CuSO}_{4} \cdot 5 \mathrm{H}_{2} \mathrm{O}$ ), $\mathrm{Mn}^{++}$(as $\left.\mathrm{MnSO}_{4} \cdot 4 \mathrm{H}_{2} \mathrm{O}\right)$ and $\mathrm{Zn}^{++}\left(\right.$as $\left.\mathrm{ZnSO}_{4} \cdot 7 \mathrm{H}_{2} \mathrm{O}\right) 2 \mu \mathrm{M}$. each when used, but they were usually omitted in experiments with a high $\mathrm{Ca}^{++}$concentration. In general, we found it best to use all six metals. The media were buffered with 30 mm. phosphate, pH 7.6, prepared from thrice-recrystallized $\mathrm{KH}_{2} \mathrm{PO}_{4}$.

Conditions of cultivation. All cultures were incubated aerobically, $5 \mathrm{ml} . / 50 \mathrm{ml}$. conical flask, in an atmosphere of $5 \% \mathrm{CO}_{2}+95 \%$ air. They were left stagnant, since shaking, before the appearance of visible growth, was inhibitory.

Measurement of growth. Viable counts were used since turbidimetric methods were unreliable. Counts on cultures showing visible growth were carried out 
either at $24 \mathrm{hr}$. intervals or, for some experiments at $36^{\circ}$ where the rise and fall in count might occur within this period, at $12 \mathrm{hr}$. intervals. Cultures showing no visible growth were usually tested weekly and incubated till no colonies were produced from $0.25 \mathrm{ml}$. of a $1 / 10$ dilution. The agar used for counts contained tryptic digest of beef, diluted to contain $0.5 \%$ total $\mathbf{N}$, together with $1.5 \%$ Oxoid (Oxo Ltd.) agar, $5 \%$ peptic digest of sheep's blood and 1/500,000 crystal violet (Herbert, 1947). Plates were dried $1 \mathrm{hr}$. at $60^{\circ}$ and inoculated with $0.25 \mathrm{ml}$. of suitable dilutions of culture, spread on the surface by means of a bent glass rod (cf. Snyder, 1947). Loss on the spreader was of the order of $1 \%$. The plates were incubated $40 \mathrm{hr}$. at $28^{\circ}$.

Using the whole surface of a $9.5 \mathrm{~cm}$. diameter plate it was thus possible to count up to 400-500 colonies/plate without observing statistical evidence for overcrowding. Usually 400-750 colonies were counted on two to four plates inoculated with serial twofold dilutions, the estimated accuracy being of the order of $\pm 13 \%(P=0.95)$. Greater accuracy was not essential, since the counts of duplicate cultures rarely showed such small differences.

Strains. In addition to the virulent strains 327, 336 and 337 and the avirulent strains Soemedang S and Tjiwidej S used by Herbert (1949), Java S, a third avirulent strain of Otten (1936) was also used.

Standardization of inocula. Tryptic beef + peptic blood agar stabs were made direct from gelatin ascorbic acid dried stock cultures (Stamp, 1947). The stabs were preserved at $0-2^{\circ}$ and subcultured as required on to tryptic beef agar slopes, to decrease the haemin content of the organisms. After incubation for 16-18 hr. at $37^{\circ}$, some of the growth was raked from the surface and emulsified in $c .5 \mathrm{ml}$. of $25 \mathrm{~mm}$. phosphate buffer. After centrifuging and resuspending the organisms in phosphate buffer, the density of the suspension was determined nephelometrically, and dilutions made so as to give an inoculum c. $5 \times 10^{3}$ organisms $/ \mathrm{ml}$. culture. The inoculum size was always checked by viable count.

\section{RESULTS}

Preliminary experiments with strain Tjiwidej $\mathbf{S}$ showed that even with an inoculum as large as $2 \times 10^{5}$ viable cells $/ \mathrm{ml}$., the minimal 8 amino-acids of Rao (1939) were inadequate for growth at $37^{\circ}$. Moderate growth was observed, however, in 4-8 days at $31^{\circ}$ when alanine and arginine were replaced by valine and isoleucine. The medium then contained: phenylalanine, valine, leucine, isoleucine, cysteine, lysine, proline. An experiment at five temperatures between 21 and $37^{\circ}$ showed that growth was most rapid at $28^{\circ}$ and that glycine, proline and lysine were not essential at this temperature. The temperature of $28^{\circ}$ was therefore chosen for further investigation, although high counts were maintained longer at lower temperatures. Subsequently, the additional nutrient requirements for growth at $36^{\circ}$ were examined.

\section{Nutritional requirements at $28^{\circ}$}

Avirulent strain Tjiwidej $S$. When the inoculum was decreased to $10^{4}$ cells $/ \mathrm{ml}$. or less, an increasingly stringent requirement for haemin was observed at $28^{\circ}$, as had been found by Herbert (1949) at $37^{\circ}$ for five strains, including Tjiwidej S. 
The only amino-acids in the medium were now phenylalanine, valine, leucine, isoleucine and cysteine. Phenylalanine was found to be absolutely essential, but in view of the inhibition of growth with some combinations of valine, leucine and isoleucine, first shown by Gladstone (1939) with Bacillus anthracis and found by Doudoroff (1943) to occur with Past. pestis, the requirement for cysteine was tested in conjunction with all combinations of these unsubstituted amino-acids. With the complete mixture growth was only moderate and not always reliable. The only other combination which supported growth was that containing valine and isoleucine, but this only did so in three out of twelve experiments.

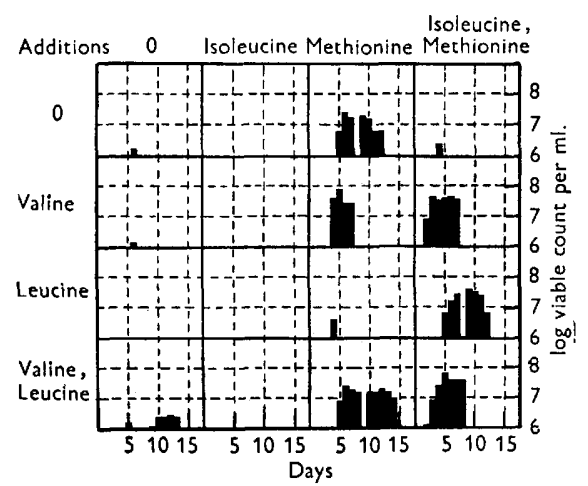

Fig. 1

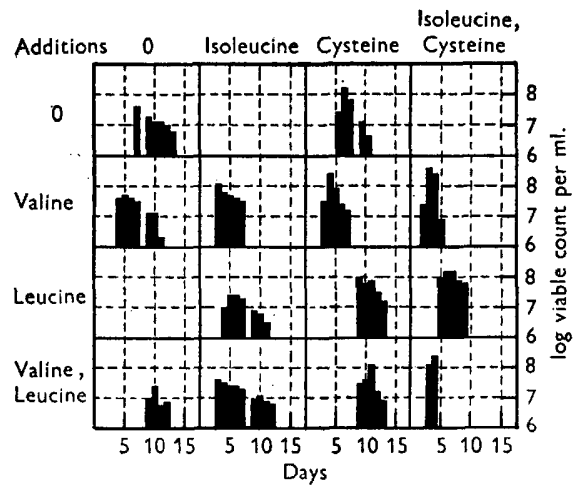

Fig. 2

Fig. 1. Inter-relationships between requirements for valine, leucine, isoleucine and methionine at $2^{\circ}$. Strain: Tjiwidej S. Basal medium: phenylalanine, haemin. No counts were made at 8 days.

Fig. 2. Inter-relationships between requirements for valine, leucine, isoleucine and cysteine in the presence of methionine at $28^{\circ}$. Strain : Tjiwidej S. Basal medium: phenylalanine, methionine and haemin. No counts were made at 8 days.

Investigation of other sources of sulphur failed to confirm the findings of Doudoroff that methionine was not only ineffective, but even inhibited the utilization of cysteine. We found, on the contrary, that after some delay methionine and phenylalanine alone supported moderate growth, which was only slightly improved by the addition of valine and was inhibited by the separate additions of leucine and isoleucine (Fig. 1). The addition of valine plus isoleucine, however, gave markedly earlier growth.

When the methionine was supplemented as sulphur source with cysteine, the results were even better than with methionine alone. Using these two sources of sulphur together, leucine and isoleucine again proved to be inhibitory, whilst the addition of valine plus isoleucine gave a good peak count of over - $10^{8}$ cells/ml. on the second or third day (Fig. 2). In other experiments alanine was found to be inhibitory, especially with suboptimal combinations of valine, leucine or isoleucine.

Homocystine was suitable as a substitute for methionine except in the presence of cysteine (Fig. 3). The delay in growth caused by this substitution was diminished in the presence of cystine or glutathione. Unless the source of 
sulphur is adequate for rapid growth, however, results tend to be variable; thus in the presence of phenylalanine, valine, isoleucine and cysteine, growth sometimes occurs, and sometimes fails (Fig. 3). For this reason we consider methionine and cysteine together as the most suitable source of sulphur for Past. pestis.

During these experiments the requirement for haemin and phenylalanine was tested with a variety of combinations of the other amino-acids. Growth was never observed in the absence of phenylalanine but occurred occasionally

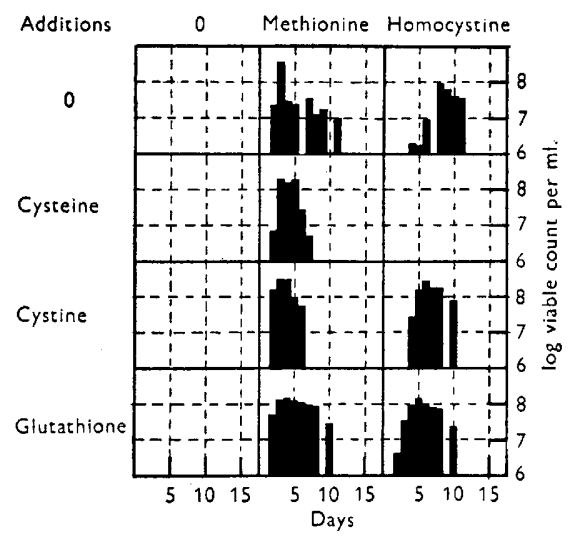

Fig. 3

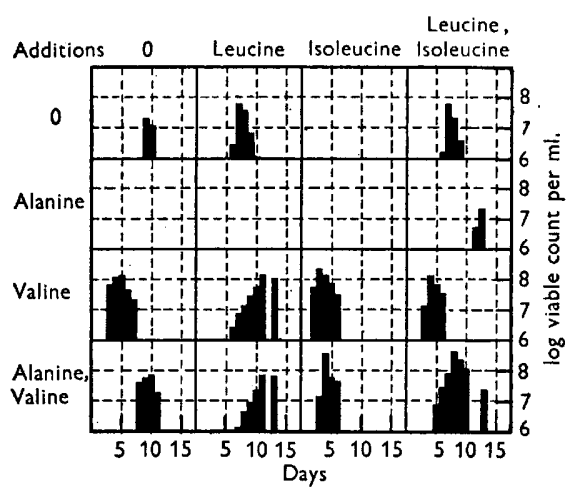

Fig. 4

Fig. 3. Sulphur requirements at $28^{\circ}$. Strain: Tjiwidej S. Basal medium: phenylalanine, valine, isoleucine and haemin.

Fig. 4. Inter-relationships between alanine, valine, leucine and isoleucine requirements at 28 . Strain: 337. Basal medium: phenylalanine, cysteine, methionine and haemin. No counts were made at 12 days.

in the absence of haemin. We also examined the effects of thiosulphate, sulphide and thiolacetate in various basal media. These compounds have been stated to act as sources of sulphur (Doudoroff, 1943), and sulphite and thiolacetate eliminate the need for haemin at $37^{\circ}$ (Herbert, 1949). At $28^{\circ}$ we have been unable to determine conditions under which either of these functions is performed consistently. When growth occurs it is invariably delayed and may be scanty. It sometimes occurs after the cell population has fallen to a few cells/ml. when chance variations in conditions may determine death or survival.

Virulent strain 337 . This grew with the same amino-acids as had been found best for Tjiwidej S, namely phenylalanine, valine, isoleucine, cysteine and methionine. Determination of the inter-relationships between the unsubstituted amino-acids showed that valine and isoleucine again formed the best combination (Fig. 4). Alanine was tested in this experiment and tended to inhibit rather than promote growth, especially in the absence of valine or isoleucine. The most satisfactory source of sulphur was again a combination of cysteine and methionine. As with strain Tjiwidej S, cystine was utilized 
as effectively as cysteine, but substitution of glutathione for cysteine or of homocystine for methionine, delayed growth for 1-4 days. The need for phenylalanine and haemin was also established.

Other strains. The four other available strains grew to a maximum population over $2 \times 10^{8}$ cells $/ \mathrm{ml}$. in $2-4$ days on the medium containing phenylalanine, isoleucine, valine, cysteine and methionine. The time taken to reach the maximum population was $\mathbf{3}$ days longer with leucine instead of valine and in one experiment Java $\mathbf{S}$ failed to grow in this medium.

\section{Nutritional requirements at $36^{\circ}$}

Avirulent strain Tjividej $S$. In the course of many experiments at $37^{\circ}$ on a medium containing 20 amino-acids similar to that of Herbert (1949), growth was frequently delayed for 5-8 days, sometimes failed and was always poor, rarely reaching $10^{8}$ cells $/ \mathrm{ml}$. Although the failures were subsequently correlated with overheating to $\mathbf{3 7 \cdot 5 ^ { \circ }}$, consistent growth was first observed as a result of adding mixtures of growth factors, of which those of the vitamin B group, especially biotin, nicotinamide, pantothenate and aneurin were of greatest importance. Temperatures above $37^{\circ}$ still caused erratic results and further experiments on the simplification of the supplements necessary for growth above $28^{\circ}$ were carried out at $36 \cdot 0 \pm 0 \cdot 5^{\circ}$.

Of the amino-acids, besides the five already mentioned as being essential, only a group containing alanine, leucine, norleucine, serine and threonine appeared to be necessary. Detailed examination of this group showed that alanine and norleucine could be omitted and that growth was delayed or much diminished in the absence of threonine. Investigation of the requirements for growth factors in the presence of this simplified amino-acid mixture (Fig. 5) showed that consistent growth was observed only with mixtures containing biotin and pantothenate together, delayed growth occurring with other mixtures or with single factors. In such cases the rate of growth was not only variable from experiment to experiment but even between duplicates in the same test. Whenever growth occurred, however, the maximum population was always of the order of $10^{8}$ cells $/ \mathrm{ml}$. Finally, the amino-acid requirements were retested in the presence of biotin and pantothenate as the only factors of the vitamin B group. Phenylalanine, cysteine, methionine, serine and threonine, and, of the unsubstituted amino-acids, valine and isoleucine, were then essential. Alanine and leucine were stimulatory.

Virulent strain 337. This strain was grown by Herbert (1949) in media containing 12-20 amino-acids at $37^{\circ}$. We confirmed his results in five out of seven experiments in which the temperature did not exceed $37^{\circ}$. In the remaining experiments, where the temperature rose to $37 \cdot 5^{\circ}$, the cultures rapidly became sterile, though growth occurred in our simplified amino-acid medium containing biotin and pantothenate. At $36^{\circ}$ the optimal combination of amino-acids was that required at $28^{\circ}$ and in addition alanine, leucine, serine and threonine (Fig. 6). Moderate growth occurred in 4-5 days, even when these latter amino-acids were omitted; leucine was the only one which by itself was stimulatory, growth occurring then after 3 days. Serine, threonine 
or alanine, added separately, were inhibitory. With serine plus threonine growth was good but delayed, this delay being overcome by the stimulatory effects of alanine and leucine together when a good peak count was obtained within 2-3 days. In the same experiment no growth occurred on omission of phenylalanine, biotin or pantothenate from the optimal medium. In other experiments the need at $36^{\circ}$ for cysteine, methionine and haemin was also confirmed. Thiosulphate, sulphite or cysteine alone did not serve as sources of sulphur either with or without haemin. The virulence of strain 337 when grown on this medium at $36^{\circ}$ was, if anything, enhanced as compared with the virulence of the same strain grown on tryptic beef broth.

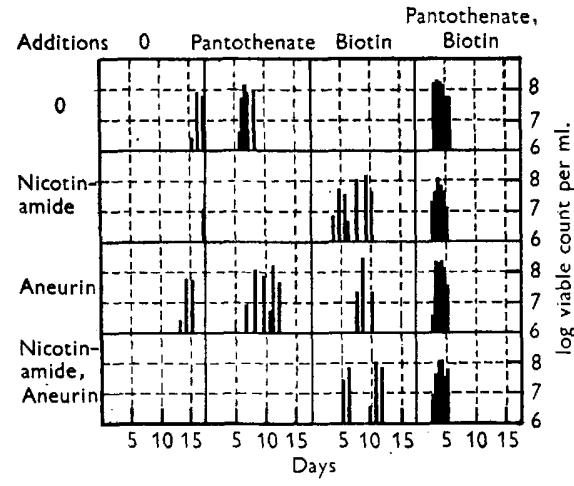

Fig. 5

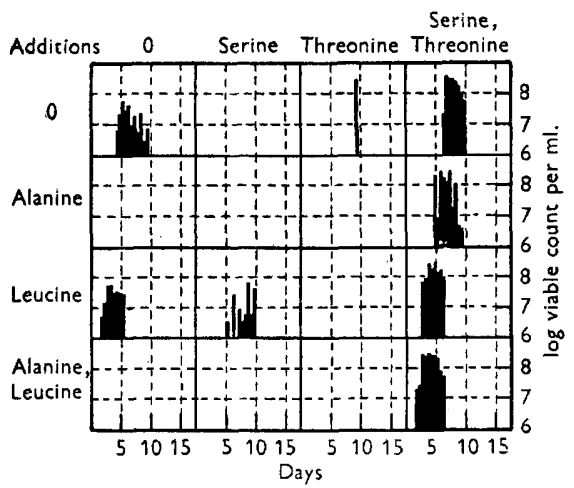

Fig. 6

Fig. 5. Requirements for biotin, nicotinamide, pantothenate and aneurin at $36^{\circ}$. Strain: Tjiwidej S. Basal medium : phenylalanine, valine, leucine, isoleucine, serine, threonine, cysteine, methionine and haemin. Two columns per day show variations between duplicate cultures. Counts were made at 8.5 days insted of at 8 and 9 days.

Fig. 6. Inter-relationships between requirements for alanine, leucine, serine and threonine at $36^{\circ}$. Strain: 337. Basal medium: phenylalanine, valine, isoleucine, cysteine, methionine, haemin, biotin and pantothenate. Two columns per day show variation between duplicate cultures.

Other strains. The other four strains available grew in our optimal medium containing phenylalanine, alanine, valine, leucine, isoleucine, cysteine, methionine, biotin, pantothenate, serine, threonine and haemin. All except Java $S$ reached a maximum population exceeding $2 \times 10^{8}$ cells $/ \mathrm{ml}$. in 2 days. The virulent strains $\mathbf{3 2 7}$ and 336 reached this count in 7 days with haemin and the 5 amino-acids, phenylalanine, valine, isoleucine, cysteine and methionine. Valine was not replaceable by leucine under these conditions.

\section{Nutritional requirements at other temperatures}

Fig. 7 shows the effect of various media on the growth of strain 337 between 23 and $\mathbf{3 6}^{\circ}$. The leucine + serine + threonine mixture was inhibitory or delayed growth at the lower temperatures though it was essential for early growth at $36^{\circ}$ in conjunction with biotin and pantothenate or 12 other amino-acids. This inhibition was also observed with strain Tjiwidej $\mathbf{S}$ but has not been further investigated. 


\section{DISCUSSION}

So far as we are aware, effects of temperature on the nutrient requirements of micro-organisms have been observed previously only with Neurospora mutants, twelve such temperature-sensitive mutants being recorded by Beadle \& Tatum (1945). Details of a mutant requiring riboflavin at $28-32^{\circ}$ have been given by Mitchell \& Houlahan (1946). Emerson (1947) described a mutant

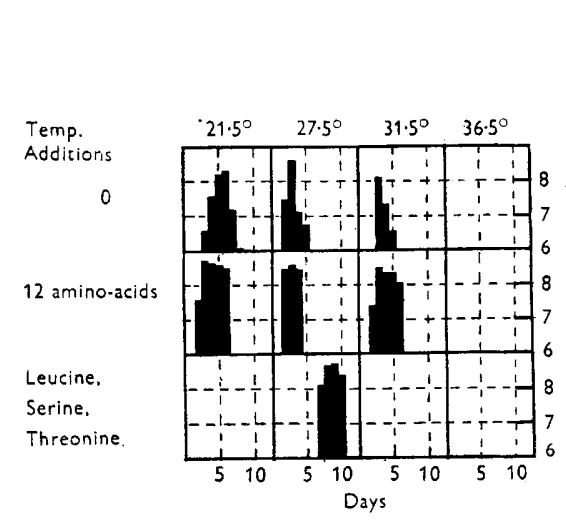

$a$

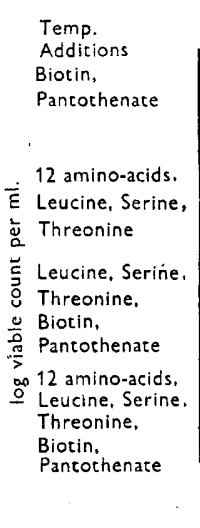

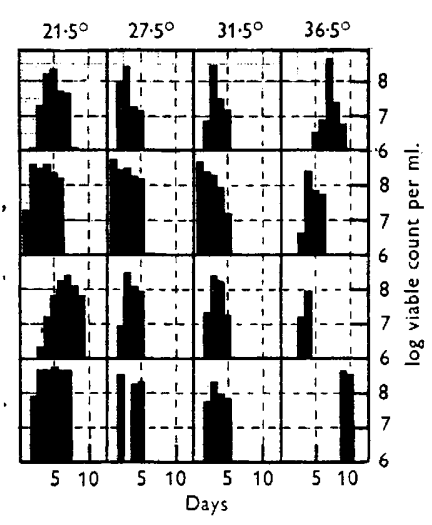

b

Fig. 7. Effect of temperature on growth in various chemically-defined media. Strain: 337 . Basal medium: phenylalanine, valine, isoleucine, cysteine, methionine and haemin. Twelve amino-acids : glycine, alanine, norleucine, aspartate, glutamate, lysine, arginine, histidine, proline, hydroxyproline, tyrosine and tryptophan. Fifteen amino-acids: 12 amino-acids, leucine, serine, threonine.

requiring sulphonamides at $35^{\circ}$ and a double mutant requiring sulphonamides and $p$-aminobenzoate at $35^{\circ}$. Such effects have not been established previously with bacteria in spite of active investigation, especially by workers with thermophilic organisms (Gaughran, 1947; Cleverdon, Pelczar \& Doetsch, 1949).

Our results at $36^{\circ}$ agree with those of other workers at $37^{\circ}$ (Berkman, 1942; Herbert, 1949) in so far as we get satisfactory growth with 20 amino-acids as source of nitrogen. We find, however, that with such a medium growth does not occur when the temperature rises to $37 \cdot 5^{\circ}$. More consistent growth is observed when 12 of these amino-acids are replaced by biotin and pantothenate. Regarding the remaining 8 amino-acids which we consider essential for satisfactory growth at $36^{\circ}$, Herbert (1949) found omission of serine or methionine to delay growth slightly, omission of threonine to delay growth markedly and omission of valine, leucine, isoleucine, cystine or phenylalanine to prevent growth. We have generally used cysteine instead of cystine, since synthetic DL-cysteine was available and it showed little difference in behaviour from cystine of natural origin (L), except in the presence of homocystine. At $28^{\circ}$ we found the most satisfactory medium to contain 4 of the amino-acids which Herbert (1949) found to be the more important, leucine, however, being replaced by methionine. This medium is more complex than that suggested by Doudoroff (1943), who considered only phenylalanine and a source of sulphur 
to be essential in a medium containing ammonium salts. The probable reason for this discrepancy is that Doudoroff used large inocula.

The organism has considerable synthetic ability. With small inocula results tend to be variable and it has been difficult to distinguish between essential and stimulatory amino-acids. Accordingly, we have regarded with suspicion late growth which failed to reach a maximum population of $10^{8} \mathrm{cells} / \mathrm{ml}$. We have only regarded a medium as adequate when the growth was practically as heavy (c. 2 to $6 \times 10^{8}$ viable organisms $/ \mathrm{ml}$ ) as that produced in $1 \%$ tryptic casein digest.

In this study we have established the qualitative nutritional requirements of six strains of Past. pestis, but in all probability we have not employed our growth factors in their optimal concentrations. The results obtained would permit a determination of these optimal concentrations; it is very probable that a balanced medium would support more rapid and abundant growth, and that the medium might even be further simplified.

Further work will be necessary before it is possible to explain why the organism is nutritionally more exacting at $36^{\circ}$ than at lower temperatures. In view of the connexion between biotin and the deamination of aspartate, serine and threonine (Lichstein \& Christman, 1948), it is tempting to suppose that biotin may facilitate the reverse process and catalyse the synthesis of these amino-acids. This may be true of aspartate, but is unlikely with serine and threonine, since these should be supplied for early growth at $36^{\circ}$ in the presence either of biotin and pantothenate or of a wider array of amino-acids. It is possible that the supply of biotin or the nutrients for its more rapid synthesis may even increase the need for serine and threonine by facilitating their destruction. The function of pantothenate as a co-factor in the oxidation of fragments containing $2 \mathrm{C}$ atoms, through the citric acid cycle, is rapidly becoming established, especially through the work of Lipmann and co-workers (e.g. Novelli \& Lipmann, 1950). It is possible that the primary function of biotin may also be concerned with energy production in this cycle in view of its part in oxaloacetate synthesis (Wessman \& Werkman, 1950). Work on the applicability of these ideas to the nutrition of Past. pestis may show whether the vitamins are specifically concerned in amino-acid synthesis or whether they are involved indirectly through energy yielding processes. Conversely, if additional amino-acids are supplied instead of biotin and pantothenate, it would be interesting to know whether the requirements for these two vitamins, presumably satisfied by synthesis, are comparable with those needed when the supply is exogenous.

The effects of temperature on nutritional requirements appear to us to be due to the enzymic processes which lead to the synthesis of essential metabolites being better co-ordinated at the optimum temperature. At higher temperatures, katabolic processes leading to the death of the organism are accelerated relative to synthetic processes and the organism becomes dependent on a wider range of preformed nutrients. An alternative explanation, the selection of mutants of differing requirements seems to us unlikely since those cultures which grow at $36^{\circ}$ begin to do so with little lag even though growth may be 
slow for several days, while those cultures which fail to grow rapidly become sterile. The selection of mutants is more likely in those cultures on suboptimal media at lower temperatures, in which delayed growth appears some time after the count has fallen to a few cells per $\mathrm{ml}$.

We wish to thank Dr D. W. Henderson and Dr D. Herbert for helpful discussion of this problem. Publication is by permission of the Chief Scientist, Ministry of Supply.

\section{REFERENCES}

Beadle, G. W. \& Tatum, E. L. (1945). Neurospora. II. Methods of producing and detecting mutations concerned with nutritional requirements. Amer. J. Bot. $32,678$.

Berkman, S. (1942). Accessory growth factor requirements of the members of the genus Pasteurella. J. infect. Dis. 71, 201.

Cleverdon, R. C., Pelczar, M. J. jun. \& Doetsch, R. N. (1949). The vitamin requirements of stenothermophilic aerobic sporogenous bacteria. J. Bact. 58, 523.

Doudonoff, M. (1943). Studies on the nutrition and metabolism of Pasteurella pestis. Proc. Soc. exp. Biol., N.Y., 53, 73.

Emerson, S. (1947). Growth responses of a sulphonamide requiring strain of Neurospora. J. Bact. 54, 195.

Gaughran, E. R. L. (1947). The thermophilic micro-organisms. Bact. Rev. 11, 189.

Gladstone, G. P. (1939). Interrelationships between amino-acids in the nutrition of B. anthracis. Brit. J. exp. Path. 20, 189.

Herbert, D. (1947). Streptomycin in experimental plague. Lancet, i, 626.

Herbert, D. (1949). Studies on the nutrition of Pasteurella pestis and factors affecting the growth of isolated cells on an agar surface. Brit. J. exp. Path. 30, 509.

Lichstein, H. C. \& Christman, J. F (1948). The role of biotin and adenylic acid in amino-acid deaminases. J. biol. Chem. 175, 649.

Mrtchell, H. K. \& Houlahan, M. B. (1946). Neurospora. IV. A temperature sensitive riboflavinless mutant. Amer. $J$. Bot. 33, 31.

Novelli, G. D. \& Lipmann, F. (1950). The catalytic function of co-enzyme $A$ in citric acid synthesis. J. biol. Chem. 182, 213.

OTteN, L. (1936). Immunisation against plague with live vaccine. Indian J. med. Res. 24, 73.

RAO, M. S. (1939). The nutritional requirements of the plague bacillus. Indian J. med. Res. 27, 75.

RAO, M. S. (1940a). Oxidations effected by the plague bacillus. Indian J. med. Res. 27, 617.

RAO, M. S. (1940b). Further studies on the nutrition of the plague bacillus: the role of haematin and other compounds. Indian J. med. Res. $27,833$.

Schürze, H. (1932). Studies on B. pestis antigens. III. The prophylactic value of the envelope and somatic antigens of B. pestis. Brit. J. exp. Path. 13, 293.

Schüтze, H. (1939). Studies on B. pestis antigens as prophylactic agents. Brit. J. exp. Path. 20, 235.

SNYDER, T. L. (1947). The relative errors of bacteriological plate counting methods. J. Bact. 54, 641.

Stamp, LoRd (1947). The preservation of bacteria by drying. J. gen. Microbiol. $1,251$.

Wessman, G. E. \& Werkman, C. H. (1950). Biotin in the assimilation of heavy carbon in oxalacetate. Arch. Biochem. 26, 214.

(Received 27 June 1951) 